\title{
Distinctive arterial collaterals caused by aortic occlusion in a patient with antiphospholipid syndrome
}

\author{
Shunya Kaneshita ${ }^{1,2}$, Masei Suda ${ }^{2}$, and Masato Okada ${ }^{2}$
}

${ }^{1}$ Inflammation and Immunology, Graduate School of Medical Science, Kyoto Prefectural University of Medicine, Kyoto; ${ }^{2}$ ImmunoRheumatology Center, St. Luke's International Hospital, St. Luke's International University, Tokyo, Japan
Received: February 4, 2020 Revised : February 4, 2020 Accepted: April 2, 2020

\section{Correspondence to}

Shunya Kaneshita, M.D.

Tel: +81-75-251-5505

Fax: +81-75-252-3721

E-mail: shunya-k@koto.kpu-m.ac.jp https:/orcid.org/0000-00016525-0690
A 41-year-old Japanese female smoker (20-pack-year) presented with a history of thrombocytopenia, aortic occlusion, and chronic kidney disease due to antiphospholipid syndrome (APS). She was diagnosed with APS on the basis of thrombosis and persistently tested positive for lupus anticoagulant diluted Russell's viper venom time test (initial-visit:follow-up after 3 months, 2.1:1.6), high-titer anticardiolipin antibody ( $\geq$ 120:104 U/mL), and high-titer anti-beta2-glycoprotein-I antibody ( $\geq$ 125:83.3 U/mL). She was treated with low-dose aspirin, warfarin, prednisolone $5 \mathrm{mg} /$ day, and hydroxychloroquine $300 \mathrm{mg} / \mathrm{day}$.
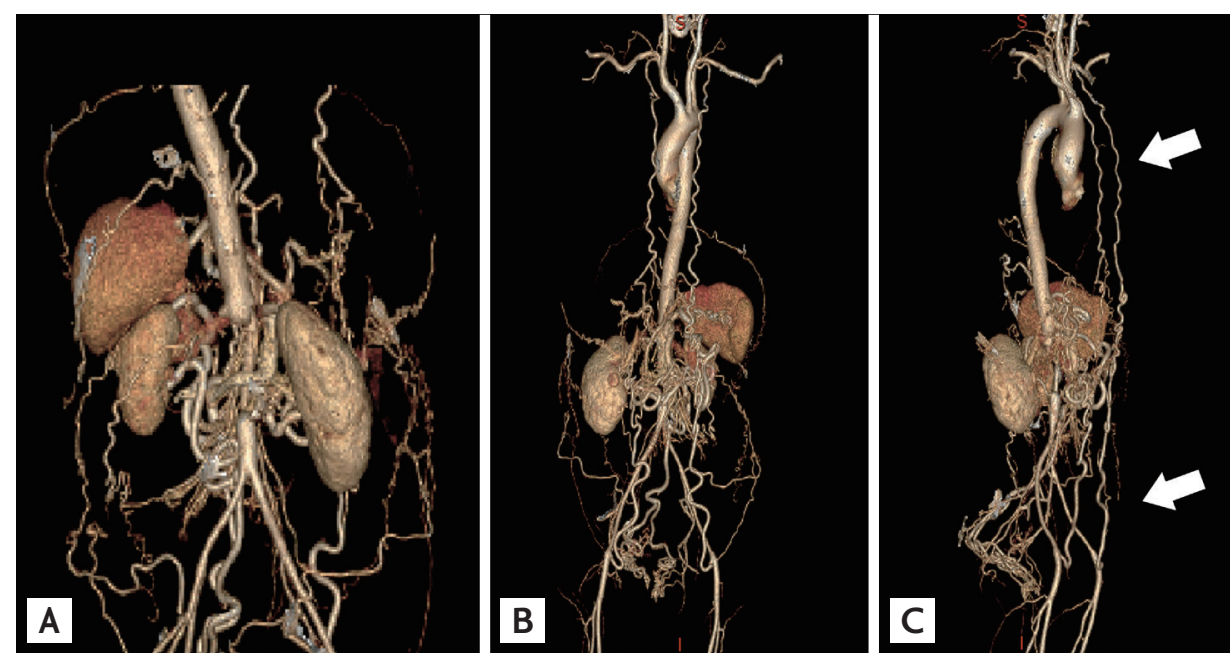

Figure 1. Three-dimensional contrast-enhanced computed tomography of the whole trunk. (A) Complete occlusion of the infrarenal aorta. (B, C) Numerous vascular collaterals and collateral arteries from the subclavian arteries to the femoral arteries on both sides (arrows). 
subsided and the ABI of the right leg improved to 0.69 upon repeated testing.

Arterial thrombosis is a common symptom among patients with APS; the thrombosis can occur at various sites such as the cerebral, renal, mesenteric, and lower limb arteries. Although emergency treatment is required in the case of acute thrombosis in these regions, it is possible that the blood flow is maintained through collateral artery development in cases of chronic throm- bosis. In such cases, the thrombosis can be managed with conservative treatment.

The patient gave written informed consent to publish the material.

\section{Conflict of interest}

No potential conflict of interest relevant to this article was reported. 